\title{
Quantify doping efficiency at the nanoscale using monochromated STEM-EELS
}

Hongbin Yang ${ }^{1}$, Andrea Konečná ${ }^{2}$, Xianghan $\mathrm{Xu}^{3}$, Sang-Wook Cheong ${ }^{4}$, F. Javier Garcia de Abajo ${ }^{5}$, Eric Garfunkel $^{6}$ and Philip Batson ${ }^{7}$

${ }^{1}$ Department of Chemistry and Chemical Biology, Rutgers University, Piscataway, New Jersey, United States, ${ }^{2}$ ICFO-Institut de Ciencies Fotoniques, United States, ${ }^{3}$ Department of physics, Rutgers University, United States, ${ }^{4}$ Rutgers Center for Emergent Materials, Rutgers University, United States, ${ }^{5}$ ICFO-Institut de Ciencies Fotoniques, Castelldefels, Catalonia, Spain, ${ }^{6}$ Department of Chemistry and Chemical Biology, Rutgers University, United States, ${ }^{7}$ Department of Physics and Astronomy, Rutgers University, United States

Doping is an important process for tailoring the electrical and optical properties of semiconductors for specific applications. Doping efficiency, defined by the ratio between charge carrier density and dopant density, is often very limited and varies significantly with the chemistry of the materials ${ }^{1}$. Despite its importance, quantifying doping efficiency usually requires multiple techniques and is only done at macroscopic scales.

Identifying individual dopant atoms has been made possible by Z-contrast imaging with aberrationcorrected scanning transmission electron microscope (STEM) ${ }^{2}$. Electron energy loss spectroscopy (EELS) can provide additional information on the location of dopants ${ }^{3}$, and is especially useful when a dopant's atomic number is close to that of the host lattice. Recently, monochromated EELS in STEM has emerged as a new way to directly probe the plasmons of free carriers in doped materials with spatial and meV energy resolution ${ }^{4}$.

In this talk, we present doping efficiency quantified by monochromated EELS in a Nion UltraSTEM 100. Carrier and dopant densities have been obtained from low-loss $(<1 \mathrm{eV})$ and core-loss EELS signals, respectively (Figure 1). Unlike the 5-50eV valence electron energy loss, both ions and free charge carriers in a solid can participate the collective excitations in the infrared spectral range. With increasing doping, properties of the free carriers dominate the low-loss dielectric response of the material. We find the doping efficiency is of order $50 \%$ for La-doped $\mathrm{BaSnO}_{3}$ (BLSO) bulk single crystals grown by the float zone method. Whereas for a Sn-doped $\mathrm{In}_{2} \mathrm{O}_{3}$ (ITO) film deposited by sputtering, the doping efficiency is no more than $30 \%$ (Figure 2). Free carrier plasmons in a freestanding ITO film will first be presented to show how we simulate the low-loss dielectric response, e.g., the surface and bulk contribution to the total energy loss, as well as the spatial resolution of the technique ${ }^{4}$. We will then discuss results on ITO and BLSO nanostructures to illustrate how the nanoscale shape and size influence the resonance energy and line width in low-loss EELS. 
(a)

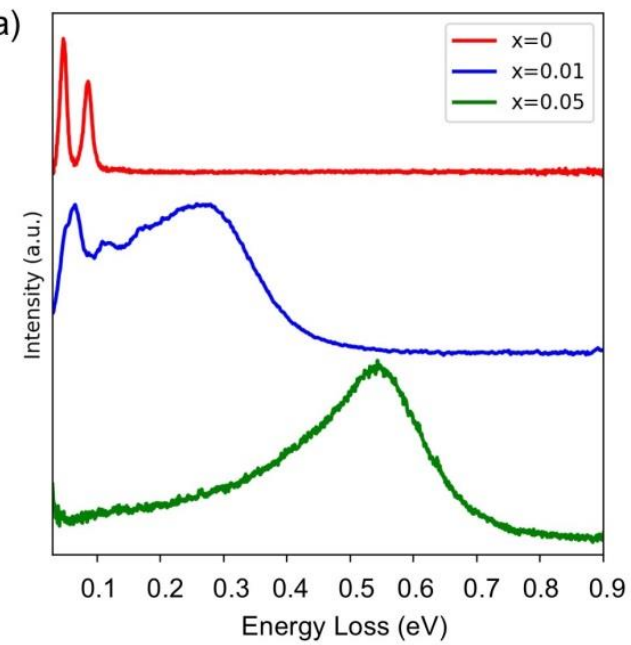

(b)

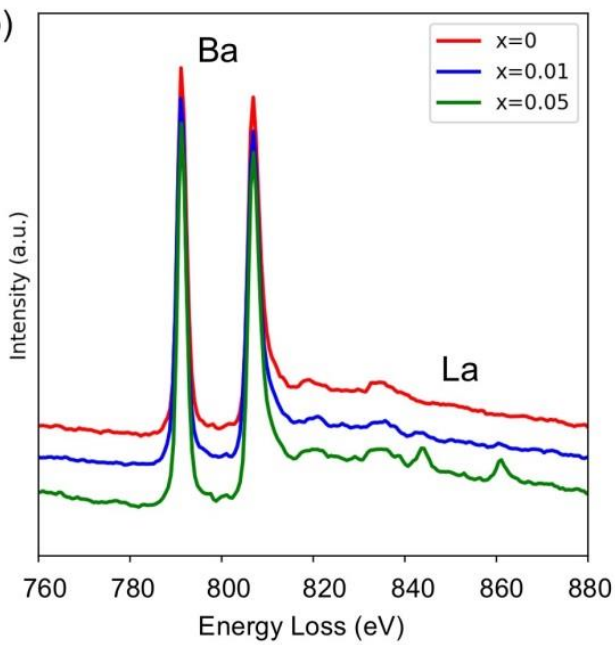

Figure 1. Figure 1. (a) Low-loss EELS acquired in the aloof geometry near Ba1-xLaxSnO3 crystals with large, cleaved surfaces. (b) Core-loss EELS showing Ba and La M4,5 edges, signals associated with La increase in intensity with doping.

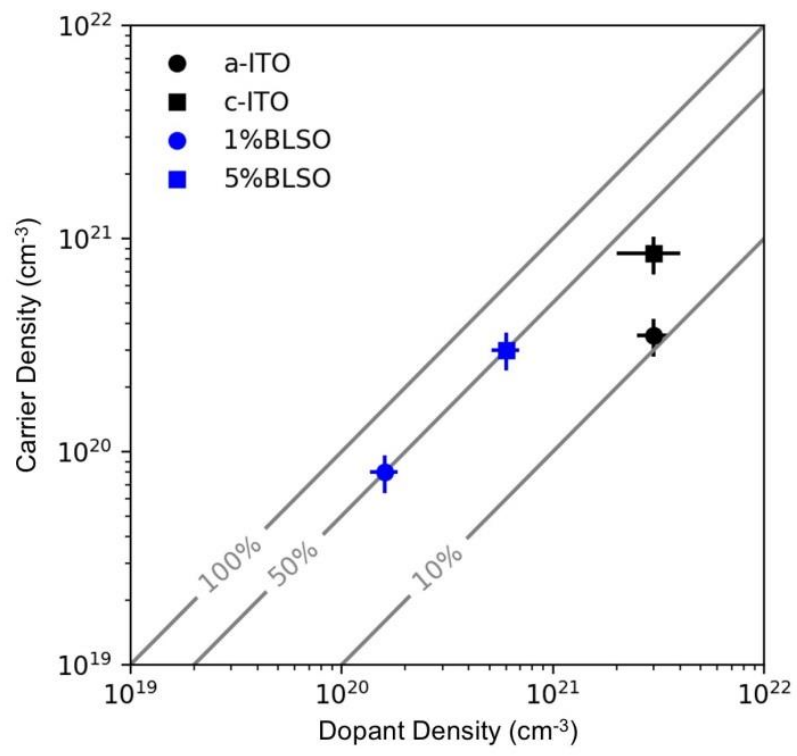

Figure 2. Figure 2. Dependence of carrier density on dopant density for ITO and BLSO. a-ITO and c-ITO indicate amorphous and polycrystalline ITO film, respectively.

References

1. G. V. Naik, V. M. Shalaev and A. Boltasseva, Adv Mater 25 (24), 3264-3294 (2013).

2. P. M. Voyles, D. A. Muller, J. L. Grazul, P. H. Citrin and H. J. L. Gossmann, Nature 416 (6883), 826829 (2002).

3. M. Varela, S. D. Findlay, A. R. Lupini, H. M. Christen, A. Y. Borisevich, N. Dellby, O. L. Krivanek, P. D. Nellist, M. P. Oxley, L. J. Allen and S. J. Pennycook, Physical Review Letters 92 (9), 095502 (2004). 4. H. Yang, E. L. Garfunkel and P. E. Batson, Phys. Rev. B 102 (20), 205427 (2020). 